\title{
UNEXPECTED RELATIONS WHICH CHARACTERIZE OPERATOR MEANS
}

\author{
HIROYUKI OSAKA AND SHUHEI WADA \\ (Communicated by Stephan Ramon Garcia)
}

\begin{abstract}
We give some characterizations of self-adjointness and symmetricity of operator monotone functions by using the Barbour transform $f \mapsto \frac{t+f}{1+f}$ and show that there are many non-symmetric operator means between the harmonic mean! and the arithmetic mean $\nabla$. Indeed, we show that there exists a non-symmetric operator mean between any two symmetric operator means.
\end{abstract}

\section{INTRODUCTION}

A bounded operator $A$ acting on a Hilbert space $H$ is said to be positive if $(A x, x) \geq 0$ for all $x \in H$. We denote this by $A \geq 0$. Let $B(H)^{+}$be the set of all positive operators on $H$ and let $B(H)^{++}$be the set of all positive invertible operators on $H$.

A real-valued function $f$ on $(0, \infty)$ is operator monotone if whenever bounded operators $A, B$ satisfy $0<A \leq B, f(A) \leq f(B)$. Functions $f(t)=t^{s} \quad(s \in[0,1])$ and $f(t)=\log t$ are typical operator monotone functions. Let $O M_{+}$be the set of positive operator monotone functions on $(0, \infty)$ and $O M_{+}^{1}=\left\{f \in O M_{+} \mid f(1)=\right.$ $1\}$.

In 6], Kubo and Ando developed an axiomatic theory for operator connections and operator means for pairs of positive operators. That is, a binary operation $\sigma$ on the class of positive operators, $(A, B) \mapsto A \sigma B$, is called a connection if the following requirements are fulfilled:

(I) $A \leq C$ and $B \leq D$ imply $A \sigma B \leq C \sigma D$.

(II) $C(A \sigma B) C \leq(C A C) \sigma(C B C)$.

(III) If $A_{n} \searrow A$ and $B_{n} \searrow B$, then $A_{n} \sigma B_{n} \searrow A \sigma B$.

A mean is a connection with normalization condition

(IV) $1 \sigma 1=1$.

Kubo and Ando showed that there exists an affine order-isomorphism from the class of operator connections onto the class of positive operator monotone functions, $\sigma \mapsto f(t)=1 \sigma(t 1)$.

This theory has found a number of applications in operator theory. In particular, Petz 9] connected the theory of monotone metrics with Kubo and Ando's operator

Received by the editors February 20, 2016 and, in revised form, June 18, 2016.

2010 Mathematics Subject Classification. Primary 47A64, 47A63.

Key words and phrases. Barbour transformation, operator monotone functions, operator connections, symmetric operator means, self-adjoint operator means.

The first author partially supported by the Program for Promotion of International Research (2014) (Ritsumeikan University).

(C)2016 by the authors under Creative Commons Attribution-Noncommercial 3.0 License (CC BY NC 3.0) 
connections. He proved that an operator monotone function $f:(0, \infty) \longrightarrow \mathbf{R}$ satisfying the functional equation

$$
f(t)=t f\left(t^{-1}\right), \quad t>0,
$$

is related to a Morozova-Chentsov function which gives a monotone metric on the Riemannian manifold of invertible $n \times n$ density matrices.

It is well known that if $f:(0, \infty) \rightarrow(0, \infty)$ is operator monotone, the transpose $f^{\prime}(t)=t f\left(\frac{1}{t}\right)$, the adjoint $f^{*}(t)=\frac{1}{f\left(\frac{1}{t}\right)}$, and the dual $f^{\perp}=\frac{t}{f(t)}$ are also operator monotone ([6]) and we call $f$ symmetric if $f=f^{\prime}$ and self-adjoint if $f=f^{*}$. It is shown in [6] that if $f$ is symmetric with $f(1)=1$, then the corresponding operator mean exists between the harmonic mean ! and the arithmetic mean $\nabla$, that is, $! \leq \sigma_{f} \leq \nabla$

In this note, we characterize symmetric functions and self-adjoint functions using Barbour transform $-: O M_{+} \rightarrow O M_{+}^{1}$ defined by $\hat{f}=\frac{t+f}{1+f}$, and characterize the class of non-symmetric operator functions and the class of operator connections $\sigma$ which satisfy $\sigma \leq$ ! or $\sigma \geq \nabla$. We show the existence of non-symmetric operator means between any pair of symmetric means.

\section{BARBOUR TRANSFORM}

In [7, for any strictly positive continuous functions $\alpha, \beta, \gamma$ on $(0, \infty)$, the Barbour path $\phi_{\alpha, \beta, \gamma}:[0,1] \rightarrow O M_{+}^{1}$ was introduced by

$$
\phi_{\alpha, \beta, \gamma}(x)=\frac{x \alpha+(1-x) \beta}{x+(1-x) \gamma}
$$

and its basic properties are elucidated. In [1, Barbour examined a function $\phi_{t, \sqrt{t}, \sqrt{t}}(x)$ which is an approximation of the power function $t^{x}$. We will denote a Barbour path $\phi_{\alpha, \beta, \gamma}(=\phi)$ such that $\phi(0)=f, \phi\left(\frac{1}{2}\right)=g, \phi(1)=h$ by the triple $[f, g, h]$.

Proposition 2.1 ([7]). For $f \in O M_{+}$, the Barbour path $\phi_{t, f, f}=\left[1, \frac{t+f}{1+f}, t\right]$ exists in $O M_{+}^{1}$.

The transform $\widehat{\sim}: O M_{+} \rightarrow O M_{+}^{1}$ defined by $f \mapsto \phi_{t, f, f}\left(\frac{1}{2}\right)=\frac{t+f}{1+f}$ plays an important role in the analysis of $O M_{+}$and we call this transform the Barbour transform.

Proposition 2.2 (cf. [7]).

(1) For $\lambda \in(0,1)$ the transform $B_{\lambda}: O M_{+} \rightarrow O M_{+}^{1}$ defined by $f \mapsto \phi_{t, f, f}(\lambda)$ is injective and $B_{\lambda}\left(O M_{+}\right)=O M_{+}^{1} \backslash\{1, t\}$.

(2) For $\lambda \in(0,1),\left\{f \in O M_{+}^{1} \mid !_{\lambda} \leq \sigma_{f} \leq \nabla_{\lambda}\right\}=B_{\lambda}\left(O M_{+}^{1}\right)$, where $A \nabla_{\lambda} B=$ $(1-\lambda) A+\lambda B$ and $A !_{\lambda} B=\left((1-\lambda) A^{-1}+\lambda B^{-1}\right)^{-1}$, and notation $!_{\lambda} \leq$ $\sigma_{f} \leq \nabla_{\lambda}$ means that $A !_{\lambda} B \leq A \sigma_{f} B \leq A \nabla_{\lambda} B$ for any positive operators $A$ and $B$.

Note that for $f \in O M_{+}, B_{\frac{1}{2}}(f)=\hat{f}$.

For $g \in O M_{+}^{1}$, we can define the inverse map `of the Barbour transform by

$$
\check{g}(t)=\frac{t-g}{g-1}
$$


then $\check{g} \in O M_{+}$. Moreover, for $\lambda \in(0,1)$ and $g \in O M_{+}^{1}$, we can define the inverse map $B_{\lambda}^{-1}(g)$ of the transform $B_{\lambda}(g)$ by $B_{\lambda}^{-1}(g)=\frac{\lambda}{1-\lambda} \cdot \check{g}$.

By a simple calculation, we have the following relations.

Lemma 2.3. For $\lambda \in(0,1)$ and $f \in O M_{+}$, we have

$$
B_{\lambda}\left(f^{\perp}\right)=B_{1-\lambda}(f)^{\perp}, B_{\lambda}\left(f^{\prime}\right)=B_{\lambda}(f)^{*}, B_{\lambda}\left(f^{*}\right)=B_{1-\lambda}(f)^{\prime} .
$$

In particular, $\widehat{f^{\perp}}=(\hat{f})^{\perp}, \widehat{f^{*}}=(\hat{f})^{\prime}$, and $\widehat{f}^{\prime}=(\hat{f})^{*}$.

We next show some properties of the Barbour transform concerning the usual order relation for operator means.

Lemma 2.4. For $f, g \in O M_{+}$and $\lambda \in(0,1)$, the following are equivalent:

(1) $f(t) \leq g(t)$ for all $t>0$;

(2) $B_{\lambda}\left(B_{\lambda}(f)\right)(t) \leq B_{\lambda}\left(B_{\lambda}(g)\right)(t)$ for all $t>0$;

(3) $B_{\lambda}(f)(t) \leq B_{\lambda}(g)(t)$ for all $t<1$ and $B_{\lambda}(f)(t) \geq B_{\lambda}(g)(t)$ for all $t>1$.

Proof. The proof follows from the equation

$$
B_{\lambda}(f)-B_{\lambda}(g)=\frac{\lambda(1-\lambda)(g-f)(t-1)}{\{\lambda+(1-\lambda) f\}\{\lambda+(1-\lambda) g\}} .
$$

In the following section, we show that the Barbour transform is a powerful tool for finding examples of non-self-adjoint operator means.

\section{Self-Adjoint means}

In [6], Kubo and Ando asked whether there exist self-adjoint operator means besides the trivial means $w_{l}, w_{r}$, the weighted geometric means corresponding to the operator monotone functions $x^{p}(0<p<1)$. Later, Hansen ([3]) gave an integral form for a strictly positive self-adjoint operator monotone function based on an exponential map.

Using the Barbour transform, we characterize the self-adjointness in $\mathrm{OM}_{+}$and give concrete examples in this section.

Proposition 3.1. Let $f$ be a positive continuous function on $(0, \infty)$ and let $\mathcal{A}=$ $\{c, c t \mid c>0\}$. The following are equivalent:

(1) $f \in O M_{+}^{1} \backslash\{1, t\}$ and $f=f^{*}$;

(2) there exists an operator monotone function $g \in O M_{+} \backslash \mathcal{A}$ such that $f=$ $\sqrt{g g^{*}}$;

(3) there exists an operator monotone function $g \in O M_{+}$such that

$$
f=\frac{t+g+g^{\prime}}{1+g+g^{\prime}} .
$$

Proof. (1) $\leftrightarrow(2)$ : Set $g(t)=\sqrt{f}(t)$. Then $g \in O M_{+} \backslash \mathcal{A}, g=g^{*}$, and $f=\sqrt{g g^{*}}$.

Conversely, if $f=\sqrt{g g^{*}}$ for some $g \in O M_{+} \backslash \mathcal{A}$, it is obvious that $f \in O M_{+}^{1}$.

$(1) \rightarrow(3)$ : Since $f \in O M_{+}^{1}$, there exists a $g \in O M_{+}$such that $f=\hat{g}$. Since $\hat{g}^{\prime}=(\hat{g})^{*}=f^{*}=f=\hat{g}$ and the Barbour transform ^ is injective, we have $g=g^{\prime}$; that is, $g$ is symmetric. 
Hence,

$$
f=\frac{t+\frac{g}{2}+\left(\frac{g}{2}\right)^{\prime}}{1+\frac{g}{2}+\left(\frac{g}{2}\right)^{\prime}} .
$$

$(3) \rightarrow(1)$ : Set $h=g+g^{\prime}$. Then $h$ is symmetric operator monotone and $\hat{h}=f$. Furthermore,

$$
\begin{aligned}
f^{*} & =(\hat{h})^{*} \\
& =\hat{h}^{\prime} \\
& =\hat{h}=f .
\end{aligned}
$$

The above result shows that we can construct a self-adjoint mean by using a symmetric mean.

Remark 3.2. Using Proposition 3.1, we can construct many examples of self-adjoint means. For example, if $g(t)=\log (t+1)$, then the corresponding operator means of functions $\sqrt{\log (t+1) / \log \left(t^{-1}+1\right)}$ and $\frac{t+\log (t+1)+t \log \left(t^{-1}+1\right)}{1+\log (t+1)+t \log \left(t^{-1}+1\right)}$ are selfadjoint.

\section{Symmetric MEAns AND NON-SYMmetric MEANS}

4.1. Symmetric means. Symmetric means have been discussed many times in the literature [4, 6, 8]. In contrast to self-adjoint means, many examples of symmetric means are known and appear in the quantum information literature [9].

Proposition 4.1. Let $f$ be a positive continuous function on $(0, \infty)$. The following are equivalent:

(1) $f \in O M_{+}$and $f=f^{\prime}$;

(2) there exists an operator monotone function $g \in O M_{+}$such that

$$
f=g+g^{\prime}
$$

(3) there exists an operator monotone function $g \in O M_{+} \backslash \mathcal{A}$ such that

$$
f=\frac{t-\sqrt{g g^{*}}}{\sqrt{g g^{*}}-1} .
$$

Proof. (1) $\leftrightarrow(2)$ : This is obvious.

$(1) \rightarrow(3)$ : Set $h=\hat{f}$. Then $h \in O M_{+} \backslash\{1, t\}$. Since $f=f^{\prime}, h^{*}=(\hat{f})^{*}=\widehat{f}^{\prime}=$ $\hat{f}=h$. Hence by Proposition 3.1 there exists $g \in O M_{+} \backslash \mathcal{A}$ such that $h=\sqrt{g g^{*}}$. Therefore

$$
\begin{aligned}
f & =\check{\hat{f}} \\
& =\check{h} \\
& =\frac{t-h}{h-1} \\
& =\frac{t-\sqrt{g g^{*}}}{\sqrt{g g^{*}}-1} .
\end{aligned}
$$
$f$.

$(3) \rightarrow(1):$ Set $h=\sqrt{g g^{*}}$. Since $h^{*}=h$ implies that $(\check{h})^{\prime}=\check{h}, f^{\prime}=(\check{h})^{\prime}=\check{h}=$ 
Proposition 4.2. Let $f$ be a positive continuous function on $(0, \infty)$. The following are equivalent:

(1) $f \in O M_{+}^{1} \backslash\{1, t\}$ and $f=f^{\prime}$,

(2) there exists an operator monotone function $g \in O M_{+}$such that

$$
f=\frac{t+\sqrt{g g^{*}}}{1+\sqrt{g g^{*}}} .
$$

Proof. This follows from the same argument as for Proposition 3.1 using the formula $(\hat{h})^{\prime}=\widehat{h^{*}}$ for $h \in O M_{+}$.

4.2. Non-symmetric means between ! and $\nabla$. It is well known that a symmetric operator mean must be between ! and $\nabla$. To show that the converse is not true, we present an algorithm for constructing a non-symmetric mean $\sigma$ such that $! \leq \sigma \leq \nabla$.

Lemma 4.3. Let $f$ be a positive operator monotone function on $(0, \infty)$ with $f(1)=$ 1. The following are equivalent:

(1) $\sigma_{\hat{f}}$ is non-symmetric mean;

(2) $f$ is non-self-adjoint.

Proof. $(2) \rightarrow(1)$ : Since $(\widehat{f})^{\prime}=\widehat{f^{*}}$, if $f$ is non-self-adjoint operator monotone and $f(1)=1, \hat{f}$ is non-symmetric; that is, $\sigma_{\hat{f}}$ is non-symmetric.

$(1) \rightarrow(2)$ : If $\sigma_{\hat{f}}$ is a non-symmetric mean, then $\hat{f} \neq(\hat{f})^{\prime}=\widehat{f^{*}}$, which implies $f \neq f^{*}$.

Lemma 4.4. If a symmetric operator mean $\sigma$ is self-adjoint, then $\sigma=\sharp$.

Proof. Let $f$ be an operator monotone function corresponding to $\sigma$. Then

$$
f(t)=t f\left(\frac{1}{t}\right)=\frac{1}{f\left(\frac{1}{t}\right)} .
$$

Hence, $f(t)=\sqrt{t}$, and $\sigma=\sharp$.

Remark 4.5. From Lemma 4.4 we know that all the following operator means are non-self-adjoint: arithmetic mean, logarithmic mean, harmonic mean, Heinz mean, and Lehmer mean [8].

Hence, we have the following result.

\section{Proposition 4.6.}

$$
\begin{aligned}
& \left\{f \mid f: \text { non-symmetric, } f_{!} \leq f \leq f_{\nabla}\right\} \\
& =\{\hat{f} \mid f: \text { non-self-adjoint, } f(1)=1\} \\
& =\{\hat{\hat{f}} \mid f: \text { non-symmetric }\} \\
& \supset\{\hat{f} \mid f: \text { symmetric, } f(1)=1\} \backslash\left\{f_{\sharp}\right\} .
\end{aligned}
$$


Proof. The first equality is clear from Lemma 4.3

It follows from $\widehat{\hat{f}^{\prime}}=(\hat{\hat{f}})^{\prime}$ and Proposition $2.2(2)$ that

$$
\left\{f \mid f: \text { non-symmetric, } f_{!} \leq f \leq f_{\nabla}\right\}=\{\hat{\hat{f}} \mid f: \text { non-symmetric }\} .
$$

Thus, we have the second equality.

From Lemma 4.4, we have

$$
\left\{\hat{f} \mid f \neq f^{*}, f(1)=1\right\} \supset\left\{\hat{f} \mid f=f^{\prime}, f(1)=1\right\} \backslash\left\{f_{\sharp}\right\},
$$

which means the last inclusion holds.

Remark 4.7. From Proposition 4.6, a non-self-adjoint positive operator monotone function $f$ with $f(1)=1$ gives a non-symmetric operator mean $\sigma_{\hat{f}}$ such that $! \leq \sigma_{\hat{f}} \leq \nabla$. For example, let $p \in\left[-1, \frac{1}{2}\right) \cup\left(\frac{1}{2}, 2\right]$ and $\mathrm{ALG}_{p}$ be the function corresponding to the power difference mean defined by

$$
\mathrm{ALG}_{p}(t)= \begin{cases}\frac{p-1}{p} \frac{1-t^{p}}{1-t^{p-1}} & t \neq 1 \\ 1 & t=1 .\end{cases}
$$

Then $\mathrm{ALG}_{p}$ is symmetric and non-self-adjoint by Lemma 4.4. Hence, $\sigma_{\widehat{\mathrm{ALG}_{\mathrm{p}}}}$ is non-symmetric. Moreover, the Petz-Hasegawa function $f_{p}$ which is defined by

$$
f_{p}(t)=p(p-1) \frac{(t-1)^{2}}{\left(t^{p}-1\right)\left(t^{1-p}-1\right)}
$$

is non-self-adjoint. Hence, $\sigma_{\widehat{f_{p}}}$ is a non-symmetric operator mean between $!$ and $\nabla$.

Later, in Section 5, we shall give an algorithm for constructing non-symmetric operator means between! and $\nabla$ (see Lemmas 5.1 and 5.2).

4.3. Non-symmetric means between symmetric means. In this section, we show the existence of non-symmetric operator means between any pair of symmetric means.

Theorem 4.8. Let $\sigma_{1}, \sigma_{2}$ be symmetric operator means. If $\sigma_{1} \leq \sigma_{2}$ and $\sigma_{1} \neq \sigma_{2}$, then there exists a non-symmetric operator mean $\sigma$ such that $\sigma_{1} \leq \sigma \leq \sigma_{2}$.

To prove the above theorem, we need the following lemma.

Lemma 4.9. Let $h_{1}$ and $h_{2}$ be self-adjoint positive operator monotone functions on $(0, \infty)$ with $h_{1} \neq h_{2}$ and $h_{1}(1)=h_{2}(1)=1$. If $h_{1}(t) \leq h_{2}(t)$ for all $t<1$ and $h_{1}(t) \geq h_{2}(t)$ for all $t>1$, then there exists a non-self-adjoint positive operator monotone function $h$ such that

$$
\begin{aligned}
& h_{1}(t) \leq h(t) \leq h_{2}(t) \quad \text { for all } t<1, \\
& h_{1}(t) \geq h(t) \geq h_{2}(t) \quad \text { for all } t>1 .
\end{aligned}
$$

Proof. Fix a non-self-adjoint mean $g$. If $h$ is an operator monotone function corresponding to $\sigma_{h_{1}}\left(\sigma_{g}\right) \sigma_{h_{2}}$ (see [6, (2.9)]), then $h$ satisfies the above inequalities.

It follows from the assumption $h_{1} \neq h_{2}$ that there exists $\delta>0$ such that

$$
(1-\delta, 1+\delta) \subset\left\{h_{1}(t) h_{2}(t)^{-1} \mid t \in(0, \infty)\right\},
$$

and

$$
g(t) \neq g^{*}(t) \text { on }(1-\delta, 1+\delta) \backslash\{1\}
$$


So there exists $t_{0} \in(0, \infty) \backslash\{1\}$ such that $g\left(h_{1}\left(t_{0}\right) h_{2}\left(t_{0}\right)^{-1}\right) \neq g^{*}\left(h_{1}\left(t_{0}\right) h_{2}\left(t_{0}\right)^{-1}\right)$. Thus,

$$
\begin{aligned}
h\left(\frac{1}{t_{0}}\right) & =h_{1}\left(\frac{1}{t_{0}}\right) g\left(\frac{h_{2}\left(\frac{1}{t_{0}}\right)}{h_{1}\left(\frac{1}{t_{0}}\right)}\right) \\
& =\frac{1}{h_{1}\left(t_{0}\right)} g\left(\frac{h_{1}\left(t_{0}\right)}{h_{2}\left(t_{0}\right)}\right) \\
& \neq \frac{1}{h_{1}\left(t_{0}\right)} g^{*}\left(\frac{h_{1}\left(t_{0}\right)}{h_{2}\left(t_{0}\right)}\right) \\
& =\frac{1}{h_{1}\left(t_{0}\right)} \frac{1}{g\left(\frac{h_{2}\left(t_{0}\right)}{h_{1}\left(t_{0}\right)}\right)} \\
& =\frac{1}{h\left(t_{0}\right)},
\end{aligned}
$$

which implies $h \neq h^{*}$.

Proof of Theorem 4.8. Let $f_{1}, f_{2}$ be positive operator monotone functions which correspond to $\sigma_{1}, \sigma_{2}$, respectively. If we define $h_{1}:=\check{f}_{1}, h_{2}:=\check{f}_{2}$, then $h_{1}, h_{2}$ satisfy the conditions appearing in Lemma 4.9. Thus, there exists a non-self-adjoint positive operator monotone function $h$ such that

$$
h_{1}(t) \leq h(t) \leq h_{2}(t) \quad \text { for all } t<1
$$

and

$$
h_{1}(t) \geq h(t) \geq h_{2}(t) \quad \text { for all } t>1 .
$$

By a simple calculation, we have

$$
f_{1}=\widehat{h_{1}} \leq \hat{h} \leq \widehat{h_{2}}=f_{2}
$$

and

$$
\hat{h} \neq\left(\widehat{h^{*}}\right)=(\hat{h})^{\prime},
$$

which means that $\sigma_{\hat{h}}$ is a desired mean.

If we define $g(t):=\frac{1+t}{2}$ in the proof of Lemma 4.9, the function $h$ which is an operator monotone function corresponding to $\sigma_{\breve{f}_{1}}\left(\sigma_{g}\right) \sigma_{\breve{f_{2}}}$ can be written as

$$
h=\left(\frac{\check{f}_{1}+\check{f}_{2}}{2}\right) .
$$

Corollary 4.10. Let $f_{1}, f_{2}$ be symmetric positive operator monotone functions on $(0, \infty)$ with $f_{1}(1)=f_{2}(1)=1$. If $f_{1} \leq f_{2}$ and $f_{1} \neq f_{2}$, then

$$
\left(\widehat{\frac{f_{1}+\check{f}_{2}}{2}}\right)=\frac{2 t\left(1-f_{1}\right)\left(1-f_{2}\right)+\left(f_{1}-t\right)\left(1-f_{2}\right)+\left(f_{2}-t\right)\left(1-f_{1}\right)}{2\left(1-f_{1}\right)\left(1-f_{2}\right)+\left(f_{1}-t\right)\left(1-f_{2}\right)+\left(f_{2}-t\right)\left(1-f_{1}\right)}
$$

is a non-symmetric positive operator monotone function between $f_{1}$ and $f_{2}$.

Example 4.11. (1) Since $f_{!}=\hat{t}$ and $f_{\nabla}=\hat{1}, \frac{\widehat{t+1}}{2}=\frac{3 t+1}{t+3}$ is a non-symmetric positive operator monotone function between $f_{!}$and $f_{\nabla}$.

(2) Since $f_{\sharp}=\hat{f}_{\sharp}$ and $f_{\nabla}=\hat{1}, \frac{\widehat{\sqrt{t}+1}}{2}=\frac{2 t+\sqrt{t}+1}{\sqrt{t}+3}$ is a non-symmetric positive operator monotone function between $f_{\sharp}$ and $f_{\nabla}$. 


\section{Application}

It is well known that a symmetric operator mean exists between $!$ and $\nabla$. To construct non-symmetric means between ! and $\nabla$, we introduced the Barbour transform in Section 2 to characterize self-adjoint means and symmetric means.

Therefore, we can systematically construct non-symmetric operator means between! and $\nabla$.

Lemma 5.1. Let $f:(0, \infty) \rightarrow(0, \infty)$ be a continuous function. The following are equivalent:

(1) $f \in O M_{+}$and $f \geq f_{\nabla}$, that is, $f(t) \geq \frac{1+t}{2}$ for $t \in(0, \infty)$;

(2) there exists an operator monotone function $g \in O M_{+} \cup\{0\}$ and non-negative real numbers $a, b \geq \frac{1}{2}$ such that $\lim _{t \rightarrow 0} g(t)=0, \lim _{t \rightarrow \infty} \frac{g(t)}{t}=0$, and

$$
f(t)=a+b t+g(t)(t \in(0, \infty)) .
$$

Proof. The implication from (1) to (2): From Löwner's Theorem (for example, see [2, Exercise V.4.9]), there exists a positive Radon measure $\rho$ on $[0, \infty]$ such that

$$
f(t)=a+b t+\int_{0}^{\infty} \frac{t(1+\lambda)}{t+\lambda} d \rho(\lambda) .
$$

We have then

$$
\begin{aligned}
& a=\lim _{t \rightarrow 0} f(0) \geq f_{\nabla}(0)=\frac{1}{2}, \\
& b=\lim _{t \rightarrow \infty} \frac{f(t)}{t} \geq \lim _{t \rightarrow \infty} \frac{f_{\nabla}(t)}{t}=\frac{1}{2} .
\end{aligned}
$$

Define a function $g$ on $(0, \infty)$ by $g(t)=\int_{0}^{\infty} \frac{t(1+\lambda)}{t+\lambda} d \rho(\lambda)$. Then we have $g \in O M_{+}$, $\lim _{t \rightarrow 0} g(t)=0$, and $\lim _{t \rightarrow \infty} \frac{g(t)}{t}=0$.

The implication from (2) to (1) is obvious.

Lemma 5.2. Let $f:(0, \infty) \rightarrow(0, \infty)$ be a continuous function. The following are equivalent:

(1) $f \in O M_{+}$and $f \leq f_{!}$, that is, $f(t) \leq \frac{2 t}{1+t} \quad(t \in(0, \infty))$;

(2) there exists an operator monotone function $g \in O M_{+} \cup\{0\}$ and non-negative real numbers $a, b \geq \frac{1}{2}$ such that $\lim _{t \rightarrow 0} g(t)=0, \lim _{t \rightarrow \infty} \frac{g(t)}{t}=0$, and

$$
f(t)=\frac{t}{a+b t+g(t)}(t \in(0, \infty)) .
$$

Proof. The lemma follows from the observation that $f \leq f_{!}$if and only if $\frac{t}{f} \geq$ $f_{\nabla}$

Remark 5.3. The followings should be well known:

(1) If $f \in O M_{+}^{1}$ and $f \leq f_{!}$, then $f=f_{\text {! }}$.

(2) If $f \in O M_{+}^{1}$ and $f \geq f_{\nabla}$, then $f=f_{\nabla}$.

Proposition 5.4. Suppose that $f \in O M_{+}$and $f \leq f_{!}$. Then $f_{!} \leq \hat{\hat{\hat{f}}} \leq f_{\nabla}$ and $\hat{\hat{\hat{f}}}$ is not symmetric.

Proof. We first consider the case $f(1)<1$. Suppose that $\hat{f}$ is symmetric. Then $(\hat{f})^{\prime}=\left(\widehat{f^{*}}\right)=(\hat{f})$. Since ${ }^{\wedge}$ is injective, $f^{*}=f$; that is, $f$ is self-adjoint. This is 
a contradiction and therefore $\hat{f}$ is not symmetric (so is $\hat{\hat{f}}$ ). If $f(1)=1$, then, by Remark 5.3, we have $f=f$ ! and $\hat{\hat{f}}$ is not symmetric.

Corollary 5.5. Let $a, b$ be non-negative real numbers greater than or equal to $\frac{1}{2}$ and assume $g \in O M_{+} \cup\{0\}$ satisfies condition (2) in Lemma 5.2. Define a function $f:(0, \infty) \rightarrow(0, \infty)$ by $f(t)=\frac{t}{a+b t+g(t)}(t \in(0, \infty))$. Then $f \in O M_{+}, f_{!} \leq \hat{\hat{\hat{f}}} \leq f_{\nabla}$ and $\hat{\hat{f}}$ is not symmmetric.

Proof. It is obvious that $f \in O M_{+}$and $f \leq f_{!}$. By Proposition 5.4, $f_{!} \leq \hat{\hat{\hat{f}}} \leq f_{\nabla}$ and $\hat{\hat{f}}$ is not symmetric.

\section{REFERENCES}

[1] J. M. Barbour, A geometrical approximation to the roots of numbers, Amer. Math. Monthly 64 (1957), 1-9. MR0086098

[2] Rajendra Bhatia, Matrix analysis, Graduate Texts in Mathematics, vol. 169, Springer-Verlag, New York, 1997. MR 1477662

[3] Frank Hansen, Selfadjoint means and operator monotone functions, Math. Ann. 256 (1981), no. 1, 29-35, DOI 10.1007/BF01450940. MR620119

[4] Fumio Hiai and Hideki Kosaki, Means for matrices and comparison of their norms, Indiana Univ. Math. J. 48 (1999), no. 3, 899-936, DOI 10.1512/iumj.1999.48.1665. MR.1736973

[5] Fumio Hiai and Dénes Petz, Introduction to matrix analysis and applications, Universitext, Springer, Cham; Hindustan Book Agency, New Delhi, 2014. MR3184500

[6] Fumio Kubo and Tsuyoshi Ando, Means of positive linear operators, Math. Ann. 246 (1979/80), no. 3, 205-224, DOI 10.1007/BF01371042. MR563399

[7] Fumio Kubo, Noboru Nakamura, Koji Ohno, and Shuhei Wada, Barbour path of operator monotone functions, Far East J. Math. Sci. (FJMS) 57 (2011), no. 2, 181-192. MR2918503

[8] Yoshihiro Nakamura, Classes of operator monotone functions and Stieltjes functions, The Gohberg anniversary collection, Vol. II (Calgary, AB, 1988), Oper. Theory Adv. Appl., vol. 41, Birkhäuser, Basel, 1989, pp. 395-404. MR1038348

[9] Dénes Petz, Monotone metrics on matrix spaces, Linear Algebra Appl. 244 (1996), 81-96, DOI 10.1016/0024-3795(94)00211-8. MR:1403277

Department of Mathematical Sciences, Ritsumeikan University, Kusatsu, Shiga 5258577, JAPAN

Department of Information and Computer Engineering, Kisarazu National College of Technology, Kisarazu, Chiba 292-0041, Japan

E-mail address: wada@j.kisarazu.ac.jp 